Lesplincipaux gisement de graphites sont ceux de Passau; Schwarzbach et Mugrau, en Bohôme; ceux de la Vloravie, de l'Autriche et de la Strrie. La Sibérie possède d'importantes mines de graphite, celles de Iéniséi, cellos des Monts Batougal découvertes en 1847 par l'ingénicur français Alibert. On trouve encore le graphite dans l'ile de Coylan. Il $y$ en a de puissants gisements dans l'Amérique du Nord, i Sturbridge (Massachussets), a Ficonderoza (New-Jersey) et à Fishkill (état de New-York) et en Californie. En France, it Chardonet (Hautes-Alpes), il y a un gisement de graphite d'une falble importanco.

Le graphite cristallise en lames hexagonales, il cst gris noirâtre, il a un éclat métallique et est doux el onctucux au toucher. Il tache les doigts et laisse sur le papier des traces d'un gris de plomb.

Sa densité est assez variable. Le graphite de Ceylan a une densité voisine de 2,25 ; celui de PAustralie, 2,66; celui de Bohème de 2,44 à 2,66 .

Après purification par la potasse en fusion el lavage aux acides, puis compressions et broyages alternos, afin d'éliminer les gaz occlus, on obtient toujours le chiffe 2,255

La quantilo de cendres renfermce dans le graphite est très variable et peut atteindre jusqu'à 60 pour 100 dans certains echantillons.

Ci-joint à titre d'exemple, cuelquos analyses de graphites effecluèes par Ch. Mène.

\begin{tabular}{|c|c|c|c|c|}
\hline Gintrintes & CInnove & Gendons & $\begin{array}{c}\text { MATHRES } \\
\text { VOLATILFS }\end{array}$ & DEX'SITE \\
\hline 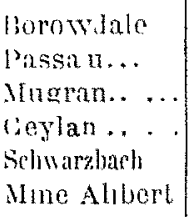 & $\begin{array}{l}91,55 \\
81,90 \\
91,05 \\
74,4 \\
88,05 \\
94,09\end{array}$ & $\begin{array}{r}7,35 \\
11,102 \\
4,85 \\
15,30 \\
10.90 \\
7,95\end{array}$ & $\begin{array}{l}1,10 \\
7,30 \\
1,10 \\
0,10 \\
1,0,5 \\
0,71\end{array}$ & $\begin{array}{l}2,3455 \\
2,3939 \\
2.1195 \\
2,3601 \\
2,1759 \\
2,1730\end{array}$ \\
\hline
\end{tabular}

\begin{tabular}{|c|c|c|c|c|c|c|c|c|}
\hline origine & $\begin{array}{l}\text { Mallèress } \\
\text { volatiles }\end{array}$ & Cendres & $\begin{array}{l}\text { Cartone } \\
\text { fixe }\end{array}$ & Si $0^{2}$ & $A A^{2} n^{3}$ & $0 e_{i}$ & $\begin{array}{c}\text { Ca } 0 \\
\text { et Mgo }\end{array}$ & Alcals \\
\hline dumberland & 1,10 & 7,35 & $91,5 \pi$ & 0,505 & $0,2 \times 3$ & 0,190 & $0.01 ; 0$ & 11019 \\
\hline Passau.. & $7,30)$ & $11,6,2$ & 81.08 & $0, \pi, 8$ & 0,356 & 0,098 & $(0,017$ & 0,020 \\
\hline Mugrau. & 4,10 & $\{, 85$ & 05 & 0,618 & $0,20,-1$ & 0,080 & 0,007 & 0,010 \\
\hline Ceylan & 5,20 & 2050 & 08,80 & 0,703 & 0,415 & 0,082 & 0,000 & 0,000 \\
\hline Austratie. & 2,15 & 72,10 & 25,75 & - & - & - & - & - \\
\hline [Brésu. & $2, \div 5$ & 20,30 & $\pi i, 1 i$ & 0.790 & 0,117 & 0,078 & 0,015 & 0,000 \\
\hline Brussin $\left(R^{n}\right)$ & $0, \pm 8$ & 7,72 & 照 & - & - & - & - & - \\
\hline Oural & 0,2 & 5,5 & 94,03 & 0612 & 0.2047 & 0110 & 0,008 & 0,003 \\
\hline Ganadu. & 1,82 & 19,70 & $i 8,48$ & $0,6.5$. & 0,901 & 0,02 & $0,0 k 5$ & 0,012 \\
\hline
\end{tabular}

Ci-joint également quelques analyses effectuées par Kern cur du graphito de Stepandvsky (Sibéric) :

\begin{tabular}{|c|c|c|}
\hline GrAPIITIS & I & II \\
\hline Caphone........... & $30,6 \mathrm{i}$ & 3940 \\
\hline shlee............ & 97,72 & 43,20 \\
\hline Sespuroxyte de fer & 402 & 3,05 \\
\hline Alumine..... & 17,80 & $1 \pi, 42$ \\
\hline chrux ot magneste & $1, \pm 0$ & 1,100 \\
\hline Malieres volitiles... & $8,2,28$ & 4,03 \\
\hline Soufre..,$\ldots . .$. & Thees & 0,04 \\
\hline
\end{tabular}

Comme on le voit, les graphites naturels sont très chargós en cendres ot demandent une purification à la fois mécanique of chimirue. On emploie dans ce but, soit les alcalis en fusion, solt les acides minéraux, tols que lacide fluorhydriquo et l'acide sulfurique.

(1 sllivre.)

Isidore BAY.

Ingénieur-Chimisle

\section{LE SERVICE D'ÉTUDE DEs GRANDES FORCES HYDRAULIQUES}

\section{PREMIERS RESULTATS}

Une ouvre dont l'mportance est capitale pour tous ccux qu1, dans notre pays, participent directement ou indrectemont i la mise en valeur de la houlle blanche, est en excellente voie de réalisation. 11 s'agit de la détermination méthodique et précise des facteurs de la puissance de nos chutes d'eau.

Pour aménager une section de rivière, il faut en connaître la pente et le débit I a détermination de la pente dépend d'un nivellement qui, tout en entraînant des frais, ne présente pas de grandes difficultés. Ma1s 1 n'en est pas de même du débit C'est l'élément de la puissance hydraulique le plus caché et le plus variable dont 1 imporie de mesurer exactement les différentes valeurs $C e$ ne sont pas seulement los débits extrêmes d'étrage ou de crues qu'on a besom de connaître, ce sont aussi les durées des divers débits intermédiaires au cours d'une année et les époques où ils se réalisient et, comme c'est surtout en hydrologre qu'1l est vra1 que les années se suivent et ne se ressemblent pas, il faut pouvorr disposer d'observations portant sur un grand nombre d'annécs pour en dégager des moyennes à peu près exactes auxquelles on puisse comparer les résultats extrêmes correspondant aux années les plus sèches et les plus humides.

L'étude méthodique des grandes forces hydrauliques devait donc avoir pour base, d'abord l'établissement d'un certain nombre de stations permanentes de jangeage dans les divers bassins de nos rivières, propres aux utılisations industrjelles. D'autre part, les débits des cours d'eau sont hés aux plures qui tombent dans leurs bassins par des lois complexes dépendant du nelief et de la nature géologique du sol. Il importe de déterminer ces lois pour chacun de nos bassins de montagne et de compléter amsi les indications des jaugeages directs, forcémont en nombre insuffisant.

Il faut donc étudier l'importance et la nature des précipitations dans chaque bassin, en même temps que par des mestres de planmétrue détaillées, l'on doit évaluer séparément. les surfaces du sol qui jouent un rôle différent dans l'alimentation des cours d'eau On se trouve ansi condult à s'occuper de pluviométrie et à planimétrer en déta1s chaque bassin.

La nécessité d'une telle étude était, depuis longtemps, reconnue. Le Congrès de la Howille blanche, tenu a Grenobic, en septembre 1902, a pris, à cet égard, une résolution bien motivée. En effet, si l'on compare l'essor de l'industrie hydroélectrique dans les Alpes françaises, suisses ou italiennes, on constate que cette industrie, née en France, attx envirous do Grenoble, des premiers essa1s de Bergès, s'y est développéc aussi rapidement qu'en aucune autre région, mais que, pat contre, la détermination exacte de la pussance hydraulique des cours d'eau est notablement moins avancée que chez nos voisins et que l'insuffisance des données sur lesquelles reposaient les premières entreprises a entraîné chez nous certains déboires. Ces considérations ont déterminé le Ministèro de l'Agriculture à organiser, par un décret en date du 25 mars I903, l'étude des questions qui se rapportent à l'évaluation des grandes forces hydrauliques en pays de montagne.

Ce Service a d'abord élé constitué dans les $\Lambda$ pes pour citre ensute étendu aux Pyrénées ct, plus tard, à l'ensemble du territoire. Son organisation et sa direction ont été confiées aux deux ingénieurs les plus compétents qui se pussent trouver en ces questions et que nos lecteurs connaissent bien : MM. René TAVERNIER et $\mathrm{R}$. DE LA BROSSE, ingénieurs en chef des Ponts et Chaussées.

Un premier rapport, que nous avons analysé très longuement dans le numéro de juillet 1906 , de La Honille Blmche, a fourni les renseignements préliminaires sur la façon dont 
ce Service est organisć, afn de poursuivre méthodiquement la réalisation d'un programme approprié et qui a pour objet mméchat : l'étude purement physique (au point de vue geo. graphique, météorologique et hydrographique) des cours d'cau ct de leurs bassins. Ce programme, inséré dans les

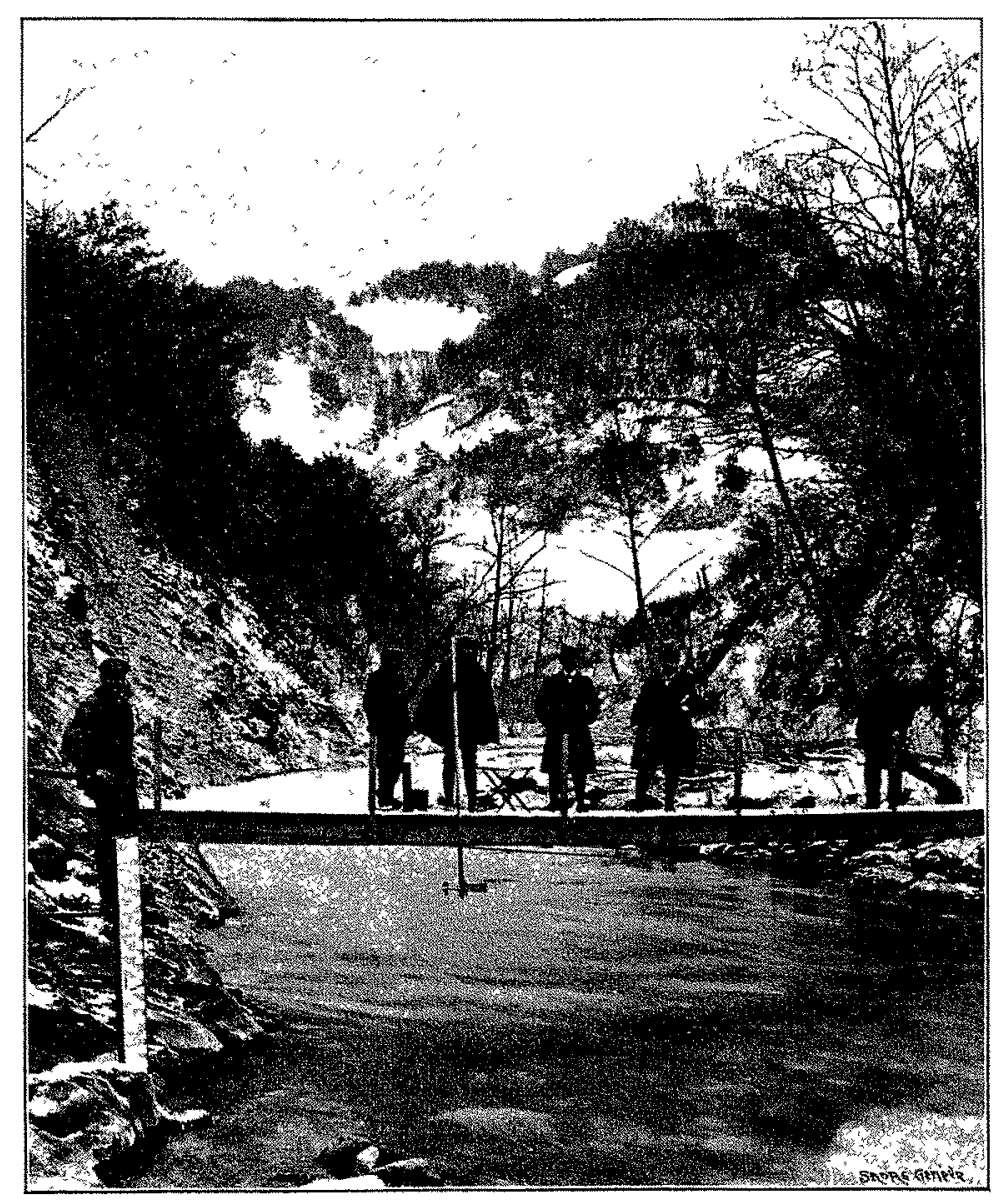

Fic. I. - Station de jaugenge de Pont-Haut, sur la Bonne (Isère), vue prise d'aval.

Anuales de la Direction de l'Hydraulaque (Ministère de l'Agriculture) comprend les chapitres suivants : I. Stations de jaugeage; Il. Pluviométrle et nivométrie; III. Planimétre; IV. Nivellements. Il indique les concours utilisés dans le fonctionnement du Service et les résultats pratiques immédiats qu'on en attend. Nous avons reproduit la carte des stations de jaugeage et des postes pluviométriques, annexée à ce rapport remarquablement intéressant.

Nous venons aujownd'hu donner l'analyse du compte-rendu des travaux du Service pendant les années 1904 et 1905 , compte rendu publié récemment par les soms de la Direction de l'Hydraulique agricole, sous la forme de deux volumes grand $1 n-8^{\circ}$ de ses Annales (1) comprenant l'un is I et l'autre 4.51 pages.

Ces volumes sont 1llustrés de nombreuses et très belles phologravures représentant l'installation des postes de jaugeages, lesquelles sont accompagnées des plans de ces stations; on y tronve les cartes en couleurs, extrêmement bien faites et très clames, tantôt au $I / 50.000^{\circ}$, tantốt à plus petite échelle des différents bassins des Alpes où se poursuivent les travaux du Service; un certain nombre de planches contiennent les graphiques des débits des principaux cours d'eau des Alpes, cnfin, quelques centaines de tableaux résument toutes les données planimétriques relatives aux divers bassins alpestres. I1 ne faut donc pas être surpris que la publication d'unc telle masse de documents n'ait pu être faute plus tôt. On aura une sdée de l'ampleur de ce travail quand on saura que ces publications contiennent notamment

(1) En vente che les éditeurs de La Houille Blanche, à Grenoble.
TOME I. - Deux rapports d'ensemble, l'un de M. de la Brosse et l'autre de M. Tavernicr, sur l'organisation des stations de jaugeage, les opérations de nuellement, les travaux de planmétrie des bassins, les ćtudes glaciares ct pluriométriques, les mesures des hauteurs d'eau et des débits des cours d'eau, les observations météorologiques relatives au Service d'étude des grandes forces hydranliques.

A la suite de ces rapports généraux vennent des rapperts spéciaux sur les bassins de l'Arve, de l'lsc̀re, de la Durance et du Var.

Ce premier volume est complété par une séric d'ćtudes, iclatives à l'installation d'une station de jaugeage, l'emplor des déversonts, la mesure des vitesses dans un comb l'cau do passage des vitesses aux débits, par MM. Taremer ct de la Brosse; la théone du tarage des moulmets, un essai de recensement provisoire des forces hydrauliques, ct l'ctude hydro logique d'un bassin de montagne par M. de la Brosse, l'oryanisation des services hydrométriques on Italic, Sursic et Bitvière, linfluence des neiges et des glacıers sur le régme des cours d'eau, une thécre du jaugeage des rivières à fond mobile, par $M$ Tavernier, étude remarquable à tous égarus et qui montre bien l'utilité des recherches théoruques scrvant à guider les obscrvations, à en limiter le nombre et à cn interpréter les résultats. L'ensemble constıtue un tranté d'hydraulique appliqué à l'étude des cours d'eau, unique cn son genre et de la plus haute utilité pour ceux qu'intéresse la mise en ouvre de la houlle blanche.

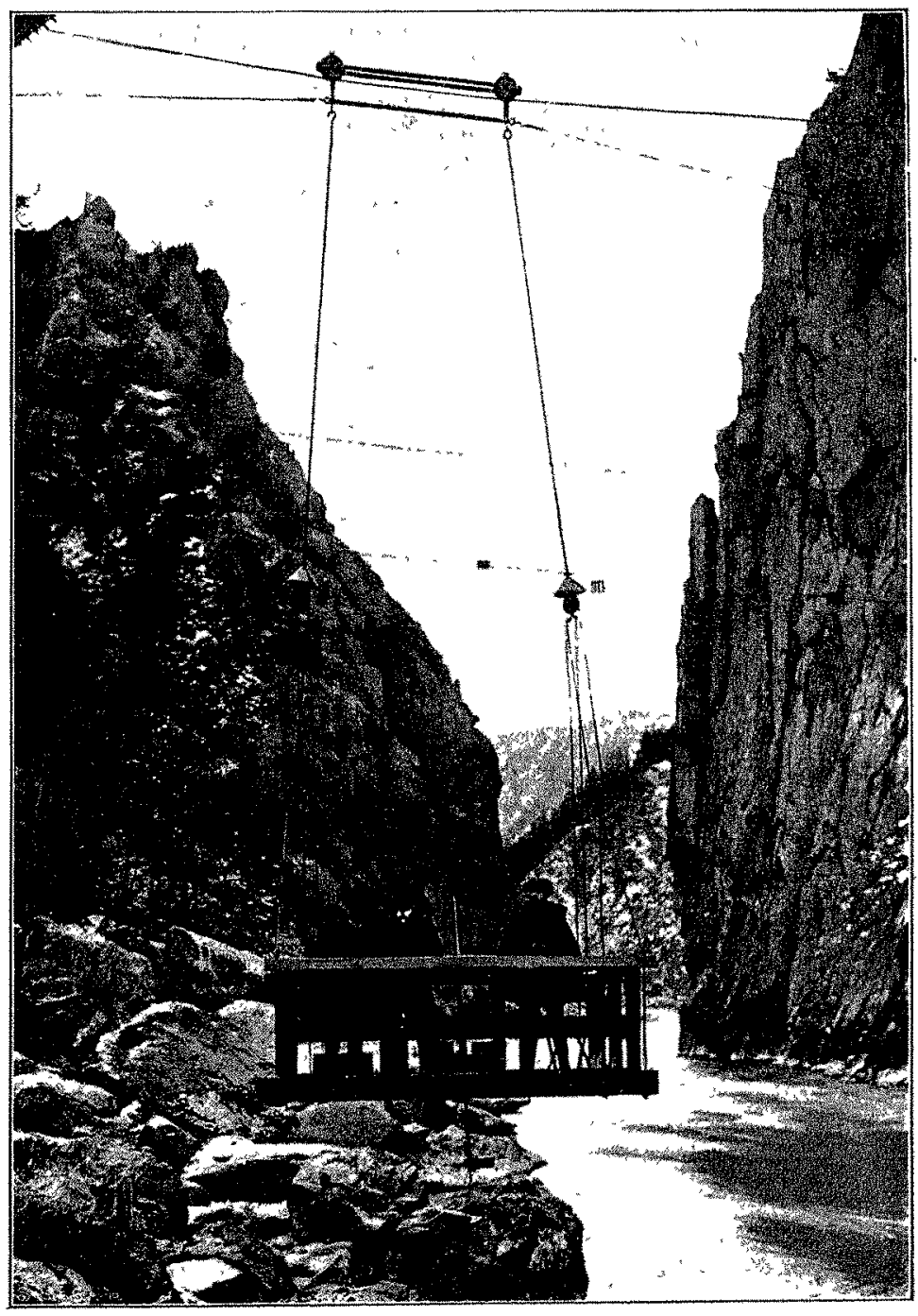

FIts. 2. - Station de jaugeage de Ponsonnas, sur le Drac; vue de la nacelle.

Sont jointes à ce volunc les cartes survantes : Deux cartes par bassins, lune de la région septentrionale des Alpes (au nord de la Durance) et lautre de la résion méridionale (Durance, Var, etc); la carte schémratique des stations de jau- 
seage et des postes pluvométriques; cufin une carte spécimen cn couleurs, au 1/50000 du bassin stupérieur de l'Arve.

TOME II. - Le deuxième volume, de $45 \mathrm{I}$ pages, grand m-8", comprend pour chacun wes bassins de T'Arve, de l'l sère, de la Durance et du Var:

I" La composition ders bassins par altitudes avec cartes génćrales en couleurs de chaque bassin;

$2^{\circ}$ Le résumé des opérations de jaugeages dans toutes les stations organisées dans chacun des bassins, et pour chaque station on $a$. les cotes et débits dos années 1904 et 1905 , la courbe des débits dédulte de ces jaugeages, les résumés du mouremont des eaux en 1904 et 1905 ;

$3^{\circ}$ I.es graphiques du régime à différentes stations de jaugeage parmi les I 50 existant alors dans les bassins étudiés.

Ces stations sont aclucllement plus nombreuses).

Les quatre cents et quelques tableaux résumant tous ces résultats sont d'une lecture très facile, leur disposition et leur classcment sont tels que, du premier coup d'œul, l'on y découvre le renselgnement que l'on pent avour à y chercher, Les courbes qui tradusent lemrs résultats sont bien nettes, ćtant tracées sur papier fort, avec mpression soignée, ce qui permet d'en étudier les noundres détauls. Les grandes carles égalment imprimées sur papier fort se prêtent, sans s'abîmer, ¿ tous les maniements désrables.

En résumé, ce deuxième volume constıtuc la plus précieuse des documentations que l'on pusse obtenir sur nos forces hydrauliques alpestres.

C'est une documentation qui se poursult et se complète chaque jour, à mesure que se recuellent les observations nouvolles. En attendant les publications ulténeures du Service l'Etudes, voyons sommaurement de quelle manière MM. de la Brossc et Taverner ont obtenu ces premiers résultats.

l.cs stations do jangeage se rattachent à plusieurs types: dles possedent toutes au moins une écholle hydrométrique dont la lecture est faite chaque jour à heure fixe (en général a inidi) par un obseriatcur du vorsmage. Plusieurs, établics sur de grands cours d'eau, sont pourvues d'un maténel de batcaux; d'autres, en plus grand nombre, ont pour base d'installation une passerelle rushque en charpente, souvent fixe (vorr figure I c1-jounte) (I), quelquefors mobile, suspendue au moyen de chaînes mouflées à un pont ou à des supports spécraux, d'autres enfin, ont reçu des dispositifs particuliers motivés par des cunditions locales exceptionnelles C'est le cas, par exemple, à Ponsonnas, sur le Drac, où l'on a dû installer une nacelle mobıle sur un câble jeté au travers d'une gorge profonde (Figures 2 et 3 ) L'organisation est à pett près complète dans la Haute-Savore, la Savore, l'Isère et les Hautes1/pes, les bassins de la. Durance et du Var; elle débute seulement dans la Drôme où les richesses hydrauliques sont, d'ailleurs, bien moins considérables.

Les instruments employés dans ces diverses stations consistent en tubes jaugeurs Darcy et en moulinetis. Quatre ou cinq stations seulement possèdent des déversoirs

Dans la majorité des cas, les jaugeages se font sur des frofils levés à chaque opération, sur lesquels on rapporte les vitesses mesurées au moyen de moulinets électriques, type Ott. Les expérimentaiteurs emploient aussı, depus quelques temps, un apparell du'même constructeur, mais plus robuste que les modèles habituels et spécialement approprié aux courants rapides ou chargés de sables. La figure 4 ci-jointe est une vue de cet instrument. 11 se distingue par la forme de sa úge qui est ovalisée avec poinlles en avant et en arrière et par la protection efficace donnée aux organes mobiles que recouve un emboitement complet. Cet appareil résiste très bien ì la flexion, même dans les courants violents où il rend de précieux services

Jusqu'à ces derniers temps, le tarage des moulmets avait lieu à Munich ou à Berne, faute de labordoires spéciaux en

(1) Nous devons i la biensellance de M. de la Brosse les photogiaphics qui ont serw ditablir ses figures.
France. MM. de la Brosse ct Tavernier sont parvenus à remédier à cette déplorable obligation en organisant une station de tarage. Elle consiste essentrellement en un manège électrique disposé sur une plateforme en charpente dans un des fossés de la fortification de Grenoble Le manège, mis cn rotation par un moteur triphasé de cinq chevaux, pent recevon des vitesses angulaures détermunées par un jeu d'engrenages interchangeables, de façon a réaliser, sur la circonférencc d'un cercle de conq mètres de diamètre, toute la série des vitesses tangentıelles depus cinq centimètres jusqu'à cinq mètres par seconde. La plate-forme curculare porte sur quatre preux entretorsés; l'arbre du manège repose sur son centrc 11 porte une sére de contacts électriques destinés à l'enregistrement sur le même graphique des nombres de tours du manège, du mouli. net expérimenté, et des secondes. I.e bras mobile, cintré de façon à dégager la plate-forme, passe au-dessus d'elle, cn

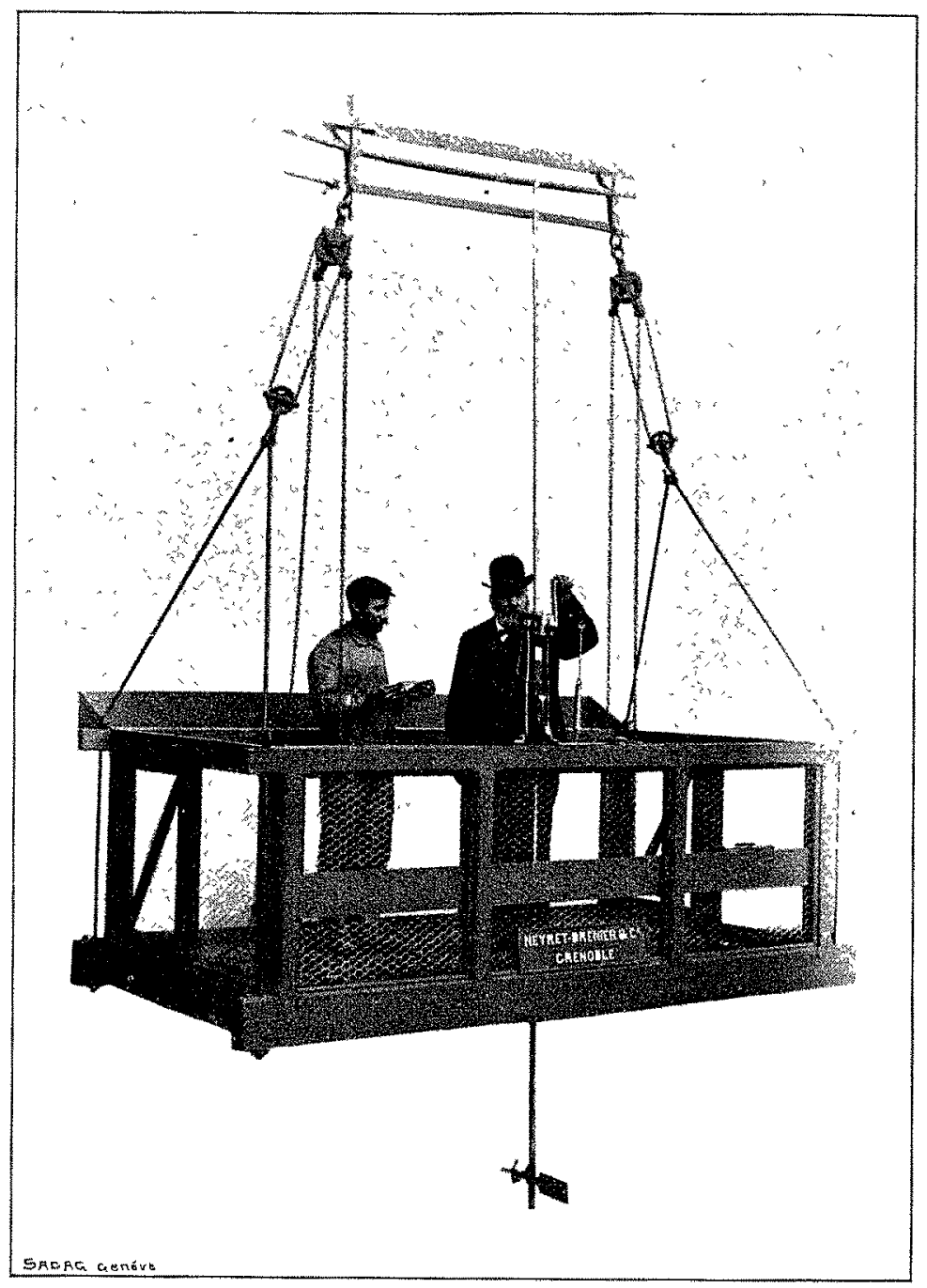

Fig 3. - Vue détallee de la nacelle portant les operateurs, suspendue dans les gorges de Ponsonnas.

laissant la place libre aux opérateurs. Il porte des douilles à vis destinées à recevorr la tige de l'instrument.

Les opérations de jaugeage, dont le nombre s'est élevé à 278 pendant les années $1904-1905$ ont pour but de trouver les courbes de débit caractéristıques de chaque station, elles sont commencées un peu partout; mais le nombre des opéraltions cst encore insuffisant pour pouvorr considérer les résultats comme définitifs Dans la majorité des cas, le lit du cours d'ean n'étant pas fixe, la mobilité du fond introdunt dans le calcul un élément varrable qui complique beaucoup la lo1 des débits. L'aticntion des opérateurs a été appelée d'une façon toute spéciale sur ces difficultés par les chefs du Service d'Etudes.

Pendant que s'effectuent les jaugeages, le Service du Nivellement général de la France, dont MM de la Brosse et Taver. 
ner ont obtenu la collaboration, complète les marlles du réseau général de nivellement. Des repères d'altitude sont scellés sur les bords immédiats des rives, soit dans le rocher, solt dans les constructions existantes à tous les confluents, prisus d'eau, ouvrages d'art, ansil qu'à tous les points intéressants accessibles, tels que chutes naturelles, changements brusques de pente, etc. Fin 1905, la longueur totale de cours d'eau ansi nrvelés dans les Alpes était de I 329 kilomètres.

Pcndant cette même année, les études glacières, commencós en 1903 et 1904 par les glaciers de Valgaudemar et de Vallouise ont été poursurvies activement sur le massif des Grandes-Rousses survant un programme combiné avec la bocićté de Géographie et l'Unıversité de Grenoble La camparne topographique qui a duré quinze jours a fourn les bases d'unc étude complète des glaciers de ce massif. Depus roob, le Comité d'études scientihques créé à la Direction de l'Hy-

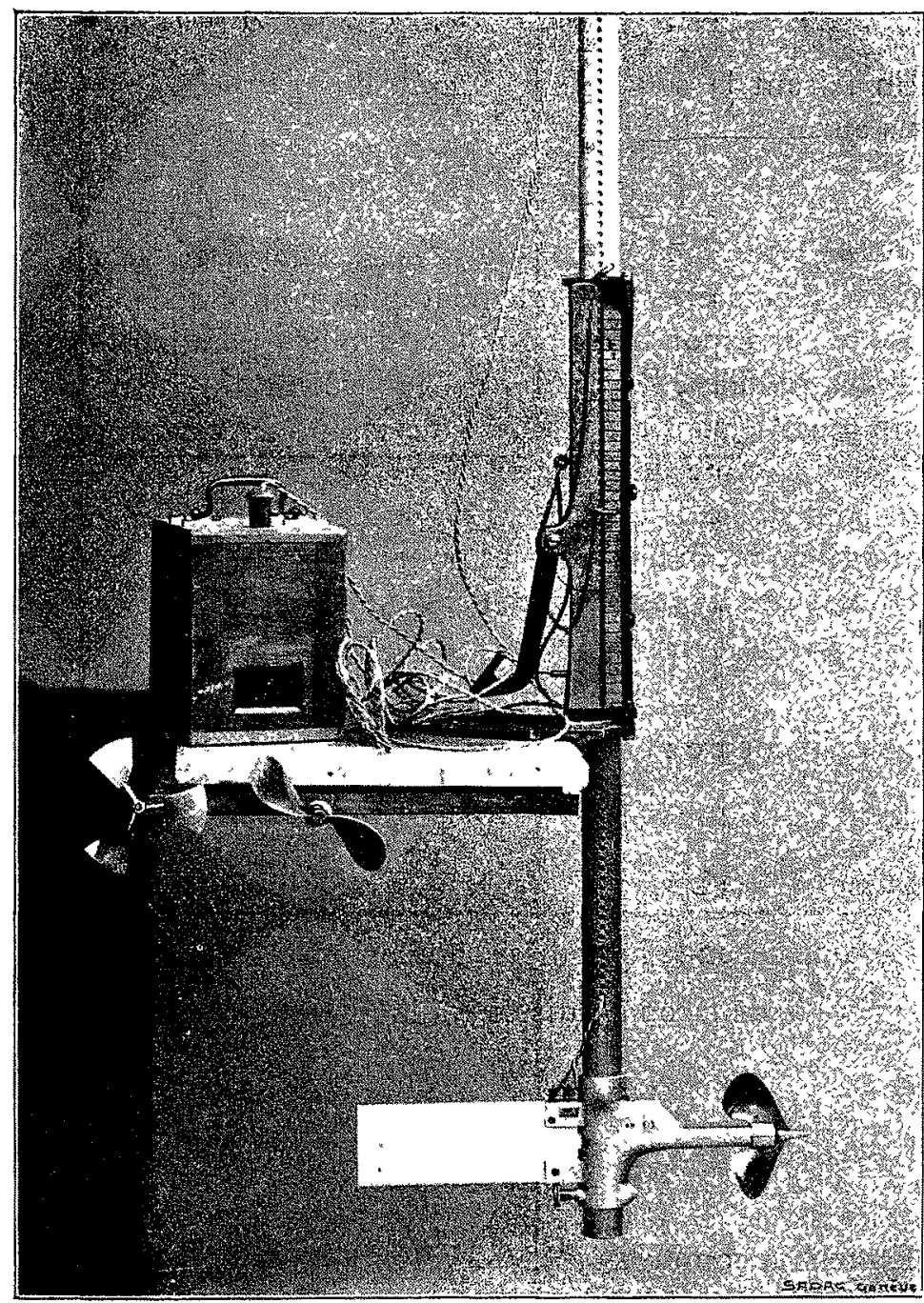

Fis. 4. - Moulbnet clectrque $\mathrm{Ctt}$ ì thge ovaliséc, avec son support, la pile de sonnene et ses hélices de rechange.

dratulique agricole, a imprimé à ces études glaciaires, une très cflicace impulsion dont les résultats ne tarderont pas à être hautement appréciés.

Le nombre des postes pluviométriques a été sensiblement augmenté, dans l'Isère, il a pasé de 24 en 1902 , à 46 en 1905 . 11 est de 40 en Savoie, y compris treize postes militaures des régions élevées, de 26 en Haute-Savore, de 6 dans les HantesAlpes et de I4 dans la Drôme le termitore sur lequel portent les études du Service étaut pourvu en 1905 de 162 postes pluvométriques. Ce nombre scra cncore accru car il reste asse" inférieur ì colu qu'on relève en Suisse par exemple, pour des surfaces équivalentes.

la planmétne des bassms est faite au moyen de truages à part sur papier fort indéformale des cartes d'Etat-Major au $1 / 50000^{\mathrm{e}}$. On y rapporte d'abord les courbes d'altitude de
500 en 500 mètres, puis les limites de chaque bassm arec les clivisions intéressant les affluents, les stations de jaugease ct les grandes prises deau. Chaque élément de surface ansi constrlué est mesuré au planmètre, plusicurs fors, par des opérateurs différents, de manière à écarter les crreurs, pur décomposé en zones d'altitude suvant les courbes de nu au tracées au préalable, les tolaux sont cnsule préparés par groupes, pus par bassin et le tout transformé cn tablcaux quu font ressortir la composition détallée de chacun d'eux ct ile ses divers éléments Le Serice d'Etudes a foums, en 1905. aux Mmisteres intéressés, les cartes au $1 / 50000^{\circ}$ des bassuns de l lisère, de l'Arve, de la Durance et du Var qu représentent, au total, 3 1.048 kilomètres carrés de bassms plammétrés par zone d'altitude. Ce traval est définitif ct ne subra que les corrections corrélatuves à la réfection des cartes d'Etat-Major Sur les cartes amsi préparées, les stations de jaugeage et le postes pluriometriques sont indiqués par des signes conventionnels, les grandes usmes hydrauliques y sont représentécs par des cexcles rouges dont lo centre est à l'cmplacencht même de l'usine et dont la surface est proportionnelle il l. pussance hydratique de l'étrblissement. A coté de chaque cercle, on trouve l'indication de la puissance accompagnéc rac signes conventionnels renseignant sur los cmplors de colte puissance.

Depus 1906, toutes les opérations du Servec se poursuncut régulièrement de manière que l'on pourra bientôt, par de nom. velles publications, comaitre tous les éléments rolatifs à l'éviluaion de nos forces hydrauliques

Comme on peut s'en convancre par cetle courte analyse des rapports de $\mathrm{XM}$ de la Brosse et Tavermer, leur princupale préoccupation a été d'mprumer à leurs recherches un arartiore pratique permettant d'utiliser de sute les résultats sous les réserves, bien entendu, que comportent le caractère alpuroximatif et le nombre encore restremt des operations effectuces Les études sont laboneuses, elles entrainent des déplacenents incessants, une correspondance considérable et comportent des détals multiples On ne saurat done trop loner les chels de ce Serrice d'Etudes de 1 admuble organisation quils ont su lu donner et qui nous valut déjà de si précieux résuliats. louange d'autant plus mérutéc que l'on peut ctre sûr qu'is dispocent de crédits très parcimoneuscment comptés ll rst donc fort désrable, dams I mécét de la précision de ces dudes comme dans colu de leur prompt achèrement, que l'Admmistration veulle bien lcur fournur les ressources nécesbal res pour les mener à bonne fin.

E. H. C otl:

\section{NOUVELLE THÉORIE DES TURBINES}

Par le Proftsseur Dr H. Loken/, de Dancig

(Sinle? ef $[n n)$

\section{IV. - Les turbines Francis.}

L'étude des turbines mixtes, ou turbines helico-centnpites, imaginées par l'américan Erancis, ct qui ont reçu tant d'applications dans ces dernières années, peut aussi se hare avec notre schéma d'écoulement:

$$
\psi=A r_{i}^{2} \quad w_{r}=-A r \quad m_{i} \quad 2.1 \%
$$

it suffit de retourner la figure suivant la figure 13, et de choisir la fonction $w_{n}$ de façon à tenir compte des variations correspondantes de la composante tangentielle w wavec les distances $r$ à l'axe, et les hauteurs - Pour réaliser la condition déja admise pour les turbines paralleles que tous les éléments des aubes dcivent exercer sur l'eau la même 